\title{
Vécu Des Enfants Soldats Pendant La Guerre: Une Analyse À Partir Des Projets De l'Organisation Non Gouvernemental Caritas Makeni En Sierra Léone
}

\author{
Dr Gnamien Yawa Ossi
}

Ecole Normale Supérieure, Département des Sciences de l’Education

doi: 10.19044/esj.2016.v13n2p256 URL:http://dx.doi.org/10.19044/esj.2016.v13n2p256

\begin{abstract}
The phenomenon of «child soldier» has become very rampant these last years. In Africa, they are very common in the Democratic Republic of Congo (DRC), in Chad, in Uganda, in Sierra Leone, and in Côte d'Ivoire. More recently, they are in Mali and in the Central African Republic. However, armed conflicts have imposed various situations on populations. The children are the main victims. The focus of this study is on child soldiers during times of war. The main objective of this study is to analyze the ways they join the armed groups and the difficulties of their social reintegration. The objective of this study is to analyze the recruitment conditions of children and the difficulties of social reintegration. The hypothesis postulates that there is a link between the passage of the children in armed groups and their psychosocial outcome. The study took place in Sierra Leone. The quantitative and qualitative methods were used. The results of the study show that: the mode of recruitment of children has an influence on their social reintegration. Also, the violent practices in armed conflicts are obstacles to their social reintegration.
\end{abstract}

Keywords: Child soldiers, War victims, Social reintegration, Mode of recruitment, Violent practices

\section{Résumé}

Le phénomène de l'«enfant soldat» a gagné de l'ampleur ces dernières années. En Afrique, les enfants soldats se trouvent principalement en République Démocratique du Congo (RDC), au Tchad, en Ouganda, en Sierra Léone, en Côte d'Ivoire ou plus récemment au Mali et en Centrafrique. Les conflits armés imposent diverses situations aux populations. Les enfants en sont les principales victimes. Les enfants soldats pendant la guerre font l'objet de notre étude. L'objectif de cette étude est de faire une analyse sur les conditions de recrutement des enfants et les 
difficultés de réinsertion sociale. L'hypothèse postule qu'il existe un lien entre le passage des enfants dans les groupes armés et leur devenir psychosocial. L'étude a pour site la Sierra Léone. Les méthodes quantitative et qualitative ont été utilisées. Les résultats de l'étude montrent que: - Le mode de recrutement des enfants a une influence sur leur réinsertion sociale; - Les pratiques violentes au cours des conflits armés constituent des obstacles à leur réintégration sociale.

Mots-clés: Enfants soldats, Victimes de guerre, Réinsertion sociale, Mode de recrutement, Pratiques violentes

\section{Introduction}

Un enfant est un être humain, mâle ou femelle en pleine croissance, dans une période de développement située entre la naissance et l'adolescence. L'enfant n'a pas les moyens de se protéger seul. C'est la raison pour laquelle il doit faire l'objet d'une attention particulière et a besoin d'une protection spécifique. La Convention Internationale des Droits de l'Enfant de 1989 définit de manière plus précise l'enfant comme étant: «[...] tout être humain âgé de moins de dix-huit ans, sauf si la majorité est atteinte plus tôt en vertu de la législation qui lui est applicable» (Bennouna 1989:4). Pendant les conflits armés, les droits des enfants sont tout simplement ignorés. Ils sont enrôlés lorsque les chefs de guerre pensent qu'il est nécessaire d'accroître les forces. Une méthode utilisée par les forces armées pendant les conflits armés est le «rafle»; des communautés; des villages sont attaqués par des groupes d'hommes afin de les enrôler de force. Selon Otunu (1999), la communauté internationale ne peut plus accepter que les enfants, les enfants innocents soient utilisés de façon cynique et exploités en tant qu'enfants soldats.

Le 12 février est la Journée internationale des enfants soldats, dédiée aux milliers de garçons et de filles enrôlés de force aux groupes armés. Contrairement à cette convention, de plus en plus d'enfants sont enrôlés directement dans les conflits armés au lieu de les protéger. Selon L'UNICEF (2011), les conflits armés ont tué plus de deux millions d'enfants; chaque année entre huit mille et dix mille enfants sont tués ou mutilés par des mines terrestres. Comme conséquences, de la guerre vingt millions d'enfants ont été contraints de fuir de chez eux ou déplacés dans leur propre pays; plus d'un million sont orphelins ou ont été séparés de leur famille; au moins six millions ont été grièvement blessés ou sont handicapés à vie et dix millions souffrent de sérieux troubles psychologiques; Le nombre des enfants soldats ne fait qu'augmenter avec de plus en plus de conflits armés sur le continent africain. 
Dans les conflits armés les enfants en sont les principales victimes. Il existe une pléthore de projets et programmes humanitaires et de réinsertion sociale sur le terrain. Malgré leur existence, il y a des difficultés de conception d'un cadre formel, fiable et durable de réinsertion sociale pour ces ex-combattants. Pourquoi malgré l'effort fournit sur le terrain, le problème d'insertion sociale de ces enfants soldats persiste? Plusieurs programmes de réinsertion des enfants soldats rencontrent des difficultés par manque d'informations suffisantes sur les expériences de ces enfants pendant les conflits armés.

Le symposium de 1997 sur l'enfant soldat organisé par l'UNICEF et d'autres organisations non-gouvernementales en Afrique du Sud a mis l'accent sur les principes à suivre pour éviter le recrutement des enfants et pour la réintégration sociale des anciens enfants soldats. Ce symposium définit le terme enfant soldat de la façon suivante: "'Child soldier' (...) means any person under 18 years of age who is part of any kind of regular or irregular armed force or armed group in any capacity, including but not limited to cooks, porters, messengers, and those accompanying such groups, other than purely as family members. It includes girls recruited for sexual purposes and forced marriage. It does not, therefore, only refer to a child who is carrying or has carried arms" (Symposium on the Prevention of Recruitment of Children into the Armed Forces and Demobilization and Social Reintegration of Child Soldiers in Africa, 1997:2).

Un enfant soldat est un être humain âgé de moins de 18 ans, recruté par une armée ou participant simplement à un conflit armé. Ces enfants soldats peuvent aussi occuper des fonctions de reconnaissance, d'espionnage, de sabotage, d'assistants aux postes de contrôle militaires, les filles sont aussi bien concernées que les garçons (Essiomle 2005). Les enfants qui ne portent aucune arme aussi appartiennent au groupe des enfants soldats. Dans les forces armées ils occupent des fonctions d'appui direct comme par exemple cuisinier, porteur ou coursier. Les filles participent non seulement aux entraînements et opérations militaires, mais doivent aussi faire les tâches «familiales» (lessive, les travaux de ménage, s'occuper des plus jeunes enfants). En outre elles sont victimes de viol et contraints à faire des mariages forcés (Humanium, 2016).

Le recrutement des enfants soldats va de l'adhésion volontaire à l'adhésion involontaire. D’après Brett, McCallin (1998) il y a quatre sortes de recrutement : recrutement prescrit par la loi qui est une prérogative de l'Etat (conscription); recrutement forcé (forced recruitment); adhésion volontaire (voluntary recruitment) et l'adhésion instruite (induced recruitment)

La majorité des enfants soldats sont passés par un recrutement forcé (UNICEF 2009). Plusieurs d'entre eux sont enlevés dans les orphelinats, les stades, les églises, les camps des refugiés etc. et sont torturés. Ils sont 
soumis à leurs recruteurs par des actions comme le viol, la maltraitance, les tortures et même l'assassinat d'un membre de leur famille.

Selon Essiomle (2005), certains enfants soldats sont recrutés volontairement mais à cause du sentiment de vengeance que les uns et les autres peuvent avoir dans la communauté, les enfants soldats ont peur de l'exprimer pendant les entretiens.

Les causes du recrutement des enfants soldats sont multiples. Les enfants soldats sont plus obéissants et dociles, faciles à manipuler que les adultes. Ils sont non seulement plus «inconscients» face aux dangers mais aussi ils voient moins la différence entre «absence» et «mort» (Essiomle 2005). Dans les pays en voies de développement et plus précisément en Afrique sub-saharienne, la pauvreté des parents, le manque d'accès à l'éducation et à la formation rendent les enfants plus vulnérables et sont des cibles privilégiées des groupes armés. Par ailleurs les investissements pour leur recrutement et leur formation sont moins élevés que chez les adultes (Steudtner 2000). En outre, les orphelins et enfants non accompagnés ou vivants dans un environnement familial difficile rejoignent les groupes armés pour leur survi et leur sécurité plutôt que de les affronter (Heckl 1999). De même, la vengeance, l'idéologie et l'identité communautaire peuvent aussi les influencer à faire partie des groupes armés (Missio aktuell 2001).

Aujourd'hui, la technologie des armes à feu favorise le recrutement de plus en plus d'enfants soldats, car les armes à tir rapide sont conçues de sorte qu'elles peuvent être facilement transportées et manipulées par un enfant; une seule pression sur la détente suffit pour tirer (Essiomle 2005). Les enfants soldats sont contraints à consommer de la cocaïne, de la marijuana et l'alcool pour qu'ils restent dociles et exécutent les ordres de leur commandants. Ils sont souvent à la recherche des modèles et veulent être aimés par les adultes. C'est pour cette raison qu’ils obéissent sans hésiter aux ordres de leurs supérieurs (Chapleau 2007).

Les enfants soldats sont non seulement victimes de guerre mais aussi auteurs de violence: Ils volent, assassinent sous l'effet de la drogue - ils tuent, ils posent des actes désapprouvés socialement. Bon nombre d'entre eux ne pourront jamais dire combien de personnes ils ont assassinées. S’ils n’obéissent pas aux ordres, on les aurait tués. Les nouveaux recrutés sont souvent soumis à des rituels de meurtres d'autres enfants (Chapleau 2007). Les filles doivent tuer ceux qui ont tenté de s’évader et sont souvent violées par les commandants et les autres soldats supérieurs en cas de refus (Deutsche Stiftung fuer Internationale Entwicklung 1995)

Les conséquences de la guerre sur les enfants soldats sont multiples. Fischer et Riedesser (1999) dans leurs travaux de recherche ont mis l'accent sur les effets des expériences traumatisantes sur un individu. Les enfants étant encore dans la phase de développement, réagissent autrement que les 
adultes. Ils sont sans défense et ces expériences ont un effet néfaste sur leur vie. Des chercheurs comme Akatcherian (2007); Vila (2006); Brett, McCallin (1998); Cohn, Goodwin-Gil (1997); Boothby (1996) ont axé leur recherches sur les conséquences de la guerre chez les enfants soldats. La méthode de recrutement, le type d'activité et de traitement de l'enfant (les tâches au sein de l'unité: service militaire ou civile), l'âge au moment du recrutement, la durée et situation sociale (avec ou sans les autres membres de la famille), la qualité de leurs expériences de vie et des conditions de vie avant le recrutement, le type de démobilisation ainsi que l'intégration dans la société sont autant de d' éléments qui s’influencent mutuellement quant au devenir psychosocial des enfants soldats.

Les problèmes que rencontrent les différents programmes de réintégration sociale des enfants soldats après les conflits armés sur le terrain préoccupent bon nombre de chercheurs. Ces programmes sont confrontés à plusieurs obstacles sur le terrain, tels que l'insuffisance de documents écrits sur les expériences réelles des ex-enfants soldats, le manque de ressources et de coopération des groupes.

Contrairement aux écrits précédents, cette étude a pour objectif de faire une analyse sur les conditions de recrutement des enfants et les difficultés de réinsertion sociale. Deux hypothèses sont formulées dans cette étude: - Il existe un lien entre le mode de recrutement des enfants et leur réinsertion sociale; - Les activités et pratiques menées après le recrutement des enfants ont une incidence sur leur réintégration sociale.

\section{Méthodologie}

\section{Sites et Participants à l'enquête}

L’enquête se déroule dans deux grandes villes de Sierra Léone: Makeni située au nord du pays et Freetown la capitale. L’échantillon porte sur un effectif de 300 enfants soldats soit 150 filles et 150 garçons qui sont pris en charge par l'organisation non gouvernementale Caritas Makeni. La raison qui justifie notre motivation à explorer le phénomène que représentent les enfants soldats, est que la Sierra Léone en tant que pays africain, constitue une zone charnière d'investigation pour les recherches scientifiques étant donné qu’elle a connu une guerre fratricide.

\section{Instruments de recueil des données}

Nous avons eu recours à la collecte d'informations sous forme d'entretien selon une démarche très structurée, selon laquelle le répondant a connaissance de l'ensemble du questionnaire, alors que l'enquêteur est chargé de noter ses réponses au fur et à mesure, en veillant à ce qu'aucune omission ou erreur ne survienne. L'entretien directif, où les ex-enfants soldats ont été interviewés, est une démarche très structurée où l’enfant soldat prend 
connaissance de l'ensemble du questionnaire et les réponses sont enregistrées. Les questions et réponses se suivent au fur et à mesure sans interruption.

Du fait de la situation délicate des enfants soldats tous les entretiens précédaient des conversations au cours desquelles les accords exacts sur l'endroit, le cadre temporaire et l'accueil (la prise des photos) des interviewés ont été conclus. Chez les enfants soldats, il est important de leur montrer l'empathie, la compassion afin qu'un climat de confiance mutuelle s'installe. En outre l'accent est mis sur le non-jugement après l'interview, peu importe les informations recueillies. Les interviews se déroulent non seulement à leurs domiciles, mais aussi à l'organisation de confiance Caritas Makeni et dans les centres de formation.

\section{Analyse des données}

A travers l'analyse quantitative les données mesurables sont traitées. Les données brutes recueillies sur le terrain à l'aide des questionnaires ont fait l'objet d'une codification et saisies sur l'outil informatique avec le logiciel «Excel». Toutes les réponses sont encodées et enregistrées en fonction des catégories et valeurs qui leur étaient assignées. L’analyse de contenu a permis ensuite d'interpréter les données, suivie d'analyse descriptive.

\section{Résultats}

L’examen des données recueillies ont permis d’aboutir aux résultats suivants:

\section{Modes de recrutement des enfants soldats}

Tableau 1: Enrôlement des enfants soldats

\begin{tabular}{|c|c|c|c|c|c|}
\hline \multicolumn{2}{|c|}{$\begin{array}{c}\text { Nombre d'enfants } \\
\text { soldats }\end{array}$} & \multicolumn{4}{|c|}{$\begin{array}{c}\text { Types } \\
\text { d'enrôlement }\end{array}$} \\
\cline { 3 - 6 } \multicolumn{2}{|c|}{} & Forcé & Pourcentage (\%) & Volontaire & Pourcentage (\%) \\
\hline Garçons & 150 & 112 & $74,66 \%$ & 38 & $25,33 \%$ \\
\hline Filles & 150 & 100 & $66,66 \%$ & 50 & $33,33 \%$ \\
\hline Totaux & $\mathbf{3 0 0}$ & $\mathbf{2 1 2}$ & & $\mathbf{8 8}$ & \\
\hline
\end{tabular}

Les enfants soldats sont aussi bien enrôlés par les forces de l'opposition que par les forces loyalistes. Les résultats de ce tableau prouvent que sur 150 garçons enrôlés, 112 sont recrutés de force; ce qui représente $74,66 \%$ de l'effectif et 38 garçons sont volontaires soit 25,33\%. Chez les filles sur 150 recrutées, 100 ont été enrôlées par force soit 66,66\% et 50 ont rejoint les groupes armés volontairement soit 33,33\%.

L'examen de ces résultats obtenus nous amène à constater que l'enrôlement par force des enfants est de coutume pendant la guerre. 
Tableau 2: Abandon des enfants soldats par leurs parents

\begin{tabular}{|c|c|c|c|c|c|}
\hline \multicolumn{2}{|c|}{$\begin{array}{c}\text { Enfants soldats } \\
\text { interviewés }\end{array}$} & \multicolumn{4}{|l|}{} \\
\cline { 3 - 6 } & $\begin{array}{c}\text { Enfants soldats } \\
\text { abandonnés par } \\
\text { les parents }\end{array}$ & $\begin{array}{c}\text { Pourcentage } \\
\mathbf{( \% )}\end{array}$ & $\begin{array}{c}\text { Enfants soldats } \\
\text { accompagnés }\end{array}$ & $\begin{array}{c}\text { Pourcentage } \\
\text { (\%) }\end{array}$ \\
\hline Garçons & 150 & 130 & $86,66 \%$ & 20 & $33,33 \%$ \\
\hline Filles & 150 & 140 & $93,33 \%$ & 10 & $6,66 \%$ \\
\hline Totaux & $\mathbf{3 0 0}$ & $\mathbf{2 7 0}$ & & $\mathbf{3 0}$ & \\
\hline
\end{tabular}

Parmi les 150 garçons interviewés 130 soit 86,66\% ont été abandonnés par les parents et 20 soit 33,33\% sont accompagnés. Le constat chez les filles se présente comme suit: sur les 150 filles, 140 soit 93,33\% sont abandonnés par les parents et seulement 10 sont accompagnées soit $6,66 \%$. Les enfants sont livrés a eux-mêmes et sont devenus enfants soldats par manque de protection de la part des adultes.

\section{Activités et pratiques pendant les conflits armés}

Tableau 3: Viol pendant les conflits armés

\begin{tabular}{|c|c|c|c|c|c|}
\hline \multicolumn{2}{|c|}{$\begin{array}{c}\text { Enfants soldats } \\
\text { interviewés }\end{array}$} & \multicolumn{5}{|c|}{} \\
\cline { 3 - 6 } \multicolumn{2}{|c|}{} & $\begin{array}{c}\text { Enfants soldats } \\
\text { victimes de viol }\end{array}$ & $\begin{array}{c}\text { Pourcentage } \\
\text { (\%) }\end{array}$ & $\begin{array}{c}\text { Enfants soldats } \\
\text { victimes atrocités } \\
\text { que le viol }\end{array}$ & $\begin{array}{c}\text { Pourcentage } \\
\text { (\%) }\end{array}$ \\
\hline Garçons & 150 & 100 & $66,66 \%$ & 50 & $33,33 \%$ \\
\hline Filles & 150 & 148 & $98,66 \%$ & 02 & $1,33 \%$ \\
\hline Totaux & $\mathbf{3 0 0}$ & $\mathbf{2 4 8}$ & & $\mathbf{5 2}$ & \\
\hline
\end{tabular}

Les données de ce tableau nous révèlent que parmi les 150 garçons 100 sont victimes de viol soit $66,66 \%$ et 50 sont victimes d'autres atrocités autre que le viol soit 33,33\%. Le constat est alarmant chez les filles, puisque sur 150 filles, 148 sont victimes de viol soit $98,66 \%$ et 02 ont subies d'autres atrocités que le viol soit 1,33\%. A travers ces chiffres nous constatons que le viol est utilisé comme un instrument de guerre sur ces enfants et rime leur quotidien. Aussi bien les garçons que les filles en sont victimes.

Tableau 4: Maltraitance pendant les conflits armés

\begin{tabular}{|c|c|c|c|}
\hline \multicolumn{2}{|c|}{ Enfants soldats interviewés } & \multicolumn{2}{|c|}{} \\
\cline { 3 - 4 } \multicolumn{2}{|c|}{} & $\begin{array}{c}\text { Enfants soldats victimes de } \\
\text { maltraitance }\end{array}$ & Pourcentage (\%) \\
\hline Garçons & 150 & 150 & $100 \%$ \\
\hline Filles & 150 & 150 & $100 \%$ \\
\hline Totaux & $\mathbf{3 0 0}$ & $\mathbf{3 0 0}$ & \\
\hline
\end{tabular}

Tous les enfants soldats interviewés, garçons comme filles, affirment avoir été sévèrement battus s’ils n’ont pas obéi aux ordres de leur Chef de guerre; ils ont aussi tué sous l'effet de la drogue. 
Certains sont obligés de tuer leurs propres parents ou leurs proches. Cet acte est un rite de passage. Beaucoup d'entre eux ne peuvent plus dire combien d'hommes ils ont tué.

Voici le témoignage d'un enfant soldat à cet effet Y, 11 ans: «Si je n’avais pas tué comme ils me l’ont ordonné, ils m’auraient tué. Je n’avais pas le choix.»

Tableau 5: Missions des enfants soldats pendant les conflits armés

\begin{tabular}{|c|c|c|c|c|c|}
\hline \multicolumn{2}{|c|}{$\begin{array}{c}\text { Enfants soldats } \\
\text { interviewés }\end{array}$} & \multicolumn{4}{|l|}{} \\
\cline { 3 - 6 } \multicolumn{2}{|c|}{} & $\begin{array}{c}\text { Enfants soldats } \\
\text { utilisés aux } \\
\text { fronts }\end{array}$ & $\begin{array}{c}\text { Pourcentage } \\
\text { (\%) }\end{array}$ & $\begin{array}{c}\text { Enfants utilisés } \\
\text { comme espions }\end{array}$ & $\begin{array}{c}\text { Pourcentage } \\
\text { (\%) }\end{array}$ \\
\hline Garçons & 150 & 130 & $86,66 \%$ & 20 & $13,33 \%$ \\
\hline Filles & 150 & 110 & $73,33 \%$ & 40 & $26,66 \%$ \\
\hline Totaux & $\mathbf{3 0 0}$ & $\mathbf{2 4 0}$ & & $\mathbf{5 2}$ & \\
\hline
\end{tabular}

Nous remarquons à la lecture de ce tableau que sur 150 garçons, 130 parmi eux ont participé directement au combat soit 86,66\% et 20 sont utilisés comme des espions soit 13,33\%. En outre sur 150 filles 110 étaient aux fronts soit 73,33\% et 40 sont envoyées pour espionnage soit $26,66 \%$. Nous pouvons affirmer que de plus en plus d'enfants sont impliqués directement dans la guerre.

\section{Interprétation et Discussion des Résultats}

Les deux hypothèses formulées dans cette étude: - Il existe un lien entre le mode de recrutement des enfants et leur réinsertion sociale; - Les activités et pratiques menées après le recrutement des enfants ont une incidence sur leur réintégration sociale; sont vérifiées

\section{Méthode de recrutement et réinsertion sociale}

Les travaux du symposium sur l'enfant soldat organisé par l'UNICEF et d'autres organisations non-gouvernementales (1997), de Essiomle (2005) et de Humanium (2016) entre autres ont permis de cadrer le terme «enfant soldat». La définition a permis d'une part de mettre en évidence les caractéristiques de ces enfants vulnérables qui ont été utilisés pendant la guerre; et d'autre part les travaux de Brett, McCallin (1998) ont su classer les différents types de recrutement des enfants soldats qui vont de l'adhésion volontaire à l'adhésion involontaire. Le manque d'accès à l'éducation ou à une formation, la pauvreté, la discrimination la profusion d'armes légères permettant l'utilisation par les enfants sont quelques causes favorables au recrutement des enfants soldats. (Essiomle 2005)

Il est indispensable de tenir compte de leur recrutement (forcé ou volontaire) dans les groupes armés. Il y a lieu de mettre en place une 
structure formelle selon laquelle, les ex-combattants dès leur démobilisation sont pris en charge et classés selon le mode de recrutement afin de concevoir des programmes plus adaptés pour les aider à surmonter leur traumatisme de guerre.

Le nombre d'enfants soldats tués lors des dix dernières années est estimé à plus de deux million et on compte plus de deux cent cinquante mille enfants soldats à travers le monde (World Vision 2013). Leur nombre s’accroît de jour en jour. Les enfants sont les plus vulnérables en période de guerre parce que séparés de leurs familles ils se retrouvent seuls, paniqués, perdus et sont dans le besoin. Il est difficile de connaître leur âge exact. Les droits fondamentaux de ces enfants sont bafoués et beaucoup d'entre eux sont traumatisés à cause des atrocités et cruautés commises pendant la guerre. Selon les informations données par le personnel d'encadrement la plupart des enfants soldats ont pris les armes parce qu'ils étaient abandonnés par les parents. Ils sont sans soutien, sans protection et certains d'entre eux trouvent refuge dans les groupes armés. D’autres enfants par contre parce dans la même situation sont capturés et fait soldats.

A l'instar des différents types de recrutement, il faut noter le nombre de plus en plus croissant des enfants abandonnés qui ont rejoint les groupes armés. World Vision (2013) déplorent la situation des enfants qui sont enrôlés pendant la guerre car seuls et livrés à eux-mêmes. Il y a lieu de notifier que la vigilance doit être prônée pendant les conflits armés de la part des organisations humanitaires pour la mise en place des "villages SOS enfants seuls” pour les accueillir.

\section{Activités et pratiques menées après le recrutement et réintégration sociale}

Les enfants soldats sont utilisés comme combattants en première ligne. Les forces armées privilégient principalement le recrutement des enfants soldats parce qu'ils sont faciles à convaincre de rejoindre ces groupes et à manipuler par rapport aux adultes. Les enfants sont, innocents, inconscients du danger et «coûtent moins chers» selon certains chefs de guerre en Sierra Leone. De plus, ils sont obéissants et vivent dans cette situation de guerre où personne ne s'occupe d'eux, ils voient souvent dans ce recrutement un moyen pour résoudre leurs problèmes et survivre. (Essiomle 2005) Au moment du recrutement des enfants, la dimension psychologique joue un rôle. Les chefs de guerre les emmenaient à les obéir sans condition. Les enfants soldats n’ont aucun moyen de se défendre face au danger parce qu'ils sont trop jeunes pour comprendre la situation. Les enfants soldats participent aux missions d'espionnage pour localiser les ennemis afin de les surprendre une fois qu'ils en ont l'occasion. D'après l'interview de l'organisation non gouvernementale allemande Missio (2001) avec un enfant 
soldat, lui et ses compagnons ont été envoyés en missions d’espionnage. Ils tiraient sur tout ce qui bougeait. Ils marchaient sur les cadavres. Un autre témoignage d'un ancien enfant soldat Carlos du Mozambique nous confirme les pratiques menées sur les champs de guerre : «Pendant la guerre, j'ai appris à aller sur des cadavres. J'ai appris que tout ce qui est important c'est la survie. Pour avoir quelque chose à manger, je devais indiquer à mes chefs que je n'avais peur de rien. J'ai lutté et tué, comme c'était de coutume pendant la guerre. Ce qui ne voulait pas tuer, était tué. C'était comme un passe-temps pour moi» (Efraime 1998 les propos de Carlos un ancien enfant soldat au Mozambique pendant son entretien avec Efraime, le Psychothérapeute Mozambicain).

Les enfants mêlés à des conflits armés sont confrontés à des événements qui les affectent émotionnellement et psychologiquement, tels que la mort violente d'un parent ou d'un proche; le fait d'avoir vu des êtres chers torturés ou tués; la séparation d'avec leur famille et leur communauté; aussi l'exposition aux bombardements, aux combats, et à d'autres situations mettant leur vie en danger. Les détentions, les arrestations, les enlèvements, les viols, la perturbation des études et de la vie communautaire appartient à leur vécu quotidien. En outre comme l’a constaté Bellamy (2011:11) «(...). Le nombre d'enfants gravement blessés ou invalides est trois fois supérieur, et ils sont encore plus nombreux à souffrir de maladies, de malnutrition, de violences sexuelles et des privations de l'exode. D'innombrables enfants sont confrontés à l'angoisse de perdre leur foyer, leurs biens et leurs proches. Dans de telles conditions, pratiquement toutes les constantes nécessaires à l'épanouissement des enfants sont gravement perturbées, et les dégâts psychologiques des conflits armés sont incalculables».

En outre, du fait de la guerre, une éventualité est à noter à travers le comportement des enfants soldats: plusieurs enfants ont «copié» le comportement de leurs «dirigeants ou chefs de guerre» qui étaient soit «durs», «sévères», «impitoyables», «agressifs», ou «moins durs» avec eux. Ils perdent facilement le contrôle et sont très agressifs au cours des discussions. Ils n'acceptent pas les points de vue contraires aux leurs. Essiomle (2005)

Comme Essiomle (2005) l'a évoqué dans ses recherches, tous les enfants soldats interviewés ont été maltraités par les chefs de guerre. Bon nombre d'entre eux ont été battus, mutilés et en portent encore des séquelles aujourd'hui. Ils sont contraints d'exécuter des ordres de tueries et d'assassinats sous l'effet de la drogue. Ils souffrent la plupart du temps des dépressions, ont des hallucinations et font des cauchemars sur leurs actions pendant les conflits surtout lorsqu'il s'agit de l'assassinat d'un proche. La mise en place des cellules de psychologues et psychiatriques dans chaque programme de réinsertion s’avère indispensable. 
Les enfants sont utilisés comme soldats aux fronts et sont envoyés pour des missions d'espionnage. Plusieurs reviennent sévèrement blessés. Aussi bien les garçons que les filles, ils sont recrutés pour servir dans les groupes armés. Ils sont victimes de viol et bon nombre servent d'esclaves sexuelles pour les soldats. Les chercheurs comme Akatcherian (2007); Vila (2006); Brett, McCallin (1998), Cohn, Goodwin-Gil (1997) se sont penchés sur les effets de la guerre sur les enfants soldats en énumérant plusieurs facteurs qui ont des influences mutuelles les uns sur les autres. Cette réalité contraint les programmes de réinsertion à une prise en charge médicale (physique et psychologique) complète des enfants. La communication entre les différents programme s'avèrent nécessaire voire obligatoire pour éviter des chevauchements et aussi des erreurs entre les programmes d'insertion des ex-combattants sur le terrain.

Une prise en charge psychosociale doit être élaborée individuellement et suivie sans interruption en vue d'un rétablissement sans faille.

\section{Conclusion}

L'objectif principal de cette étude est de faire une analyse sur les conditions de recrutement des enfants et les difficultés de réinsertion sociale. Il s'agit d'établir un lien entre le passage des enfants dans les groupes armés et leur devenir psychosocial. Ceci va conduire à la conception des programmes de réinsertion et de réintégration sociale plus adaptés après leur démobilisation. L'étude est menée sur la base de deux hypothèses à partir desquelles nous avons validé un certain nombre de résultats. Il a été prouvé que le mode de recrutement des enfants a une influence sur leur réinsertion sociale ainsi que les activités et pratiques menées après le recrutement des enfants ont une influence sur leur réintégration psychosociale.

En outre les conflits armés affectent les enfants à tous les niveaux, individuellement et aussi en tant que membres d'une famille et aussi d'une communauté. Les enfants sont les plus vulnérables à cause de leur manque de maturité à raisonner et à juger indépendamment. Des études sur les réactions posttraumatiques des enfants soldats en Afrique de l'ouest sont encore à leurs débuts. Témoins des atrocités les enfants soldats se retrouvent seuls après la guerre avec leurs séquelles physiques et psychologiques et sont rejetés par leur famille et leur communauté.

Beaucoup reste encore à faire pour interdire l'utilisation et le recrutement des enfants durant les conflits armés. C’est dans cette optique qu'une nouvelle campagne visant à mettre fin au recrutement et à l'utilisation d'enfants dans les forces gouvernementales et les troupes rebelles lors de conflits a été mis en place. 


\section{References:}

1. Akatcherian, C. (2007). Le pédiatre, l'enfant et la guerre. La Gazette de la Société Française d’Orthopédie Pédiatrique. 21.

2. Bellamy, C. (2011). Les enfants sont les premières victimes de la guerre. L’observateur (30).

3. Bennouna, M. (1989). La convention des Nations Unies relative aux droits de l'enfant. Annuaire Français de Droit International, XXXV. Paris: Éditions du CNRS.

4. Boothby, N. (1996). Children in War. Survival as a Collective Act. M. McCallin [Eds.]. The psychological well-being of refugee children. Research, Practice and Policy Issues 136-149. Geneva.

5. Brett, R.; McCallin, M. (1998). Children: The invisible Soldiers. 2nd edition. Stockholm: Rädda Barnen.

6. Chapleau, P. (2007). Enfants-soldats: Victimes ou criminels de guerre? Art de la guerre: Editions du rocher.

7. Cohn, I.; Goodwin-Gil, G. S. (1997). Child Soldiers. The role of children in armed conflicts. A study for the Henry Dunant Institute Geneva. Oxford: Clarendon Press.

8. Deutsche Stiftung fuer Internationale Entwicklung [Hrsg.] (1995). Bericht Kinder im Krieg. Berlin.

9. Efraime, B. (1998). Amnesty International Rundbrief (25) Juni 2000, 27.

10. Essiomle, Y. O. (2005). Psychologische Betreuung ehemaliger Kindersoldaten in Westafrika. Doktorarbeit im Fachbereich Erziehungswissenschaft und Psychologie an der Freie Universität Berlin. Berlin: Universitätsbibliothek Berlin.

11. Fischer, G.; Riedesser, P. (1999). Lehrbuch der Psychotraumatologie. 2. Aufl. Hamburg: Reinbek.

12. Heckl, U. (1999). Eine Armee entlaesst ihre traumatisierten Kindersoldaten in eine ungewisse Zukunft. Report Psychologie, 9 (674-683).

13. Missio aktuell (2001). Kindersoldaten sind Opfer. Wer sich weigert zu töten, wird erschlagen. Ein Bericht von Toni Görtz mit Fotos von Erol Gurian. Aachen: Missio.

14. Otunu, O. (1999). The child victims of war. BBC News Africa Friday, June 25, Published at 15:36 GMT 16:36 United Kingdom.

15. Steudtner, P. (2000). Die soziale Eingliederung von Kindersoldaten. Konzepten und Erfahrungen aus Mozambik. Berghof Report 6. Berlin: Berghof Forschungszentrum fuer konstruktive Konflikbearbeitung.

16. Symposium on the Prevention of Recruitment of Children into the Armed Forces and Demobilization and Social Reintegration of Child 
Soldiers in Africa (1997). Cape Town Annotated Principles and Best Practice on the Prevention of Recruitment of Children into the Armed Forces and Social Reintegration of Child Soldiers in Africa. Cape Town: Symposium on the Prevention of Recruitment of Children into the Armed Forces and Demobilization and Social Reintegration of Child Soldiers in Africa.

17. Vila, G. (2006). L'état de stress post-traumatique chez l'enfant. Journal de Pédiatrie et de Puériculture, 19 (3), 97-100.

18. UNICEF (2011). Les enfants dans les conflits armés et les situations d'urgence. New York: UNICEF.

19. UNICEF (2009). Protection de l'enfance contre la violence, l'exploitation et les mauvais traitements. New York.

20. World Vision (2013). Journée internationale des enfants soldats. Paris: World Vision.

\section{Webographie}

21. Humanium (2016). http://www.humanium.org/fr/enfant-soldat. Consulté le 25 Septembre 2016 à $11 \mathrm{~h}$. 